\title{
JOURNEY TIME ESTIMATION USING SINGLE INDUCTIVE LOOP DETECTORS ON NON-SIGNALISED LINKS
}

\author{
T. Cherrett, F. McLeod, H. Bell, M. McDonald \\ The Transportation Research Group, Department of Civil and Environmental Engineering, \\ University of Southampton, Highfield, Southampton. SO171BJ. U.K.
}

\begin{abstract}
$\underline{\text { Abstract }}$
This paper describes two techniques designed to estimate vehicle journey times on non-signalised roads using 250-ms digital loop-occupancy data produced by single inductive loop detectors. A mechanistic and neural network approach provided historical journey time estimates every 30 -seconds based on the data collected from the previous 30 -second period. These 30 -second estimates would provide the traffic network operator with immediate post-event congestion information on roads where no CCTV cameras were present.

The mechanistic approach estimated journey times every 30 seconds between pairs of detectors using a knowledge of vehicle speed derived from the loops and the distances between them.

The 30-second average loop-occupancy time per vehicle (ALOTPV), average timegap between vehicles (ATGBV) and percentage occupancy parameters derived from the inductive loops were presented to a neural network for training along with the associated vehicles' measured journey times.

The neural network was shown to consistently out-perform the mechanistic approach (in terms of the mean absolute percentage deviation from the mean measured travel time), particularly when using pairs of detectors.
\end{abstract}

Keywords: Transport, Traffic, Neural Networks. 


\section{Introduction}

Cities using signalised traffic control systems to improve traffic flow and reduce congestion nearly always do so through a traffic control centre. Such a dedicated facility houses not only the infrastructure for controlling traffic signals but also hardware which informs the operator of on-street conditions (close circuit television cameras (CCTV), speed detectors), and allows the dissemination of information to motorists (via variable message signs, local radio feeds and intelligent in-car units). At present, CCTV is commonly regarded as the primary medium for collecting reliable on-street information. CCTV however can only give the operator a snap-shot of conditions at a specific location whereas estimates of journey time for specific routes would provide a better picture of how well a city’s streets were performing.

Automatic registration plate recognition using CCTV has been successfully used to provide estimates of journey time ${ }^{1}$ where networks of cameras exist. A more cost effective option would result if similar estimates could be derived from the existing inductive loops controlling the city’s traffic signals.

The ability to estimate journey times accurately using loop detectors, depends on the particular format and aggregation level of the digital data produced. Most techniques have relied on the ability to obtain an accurate estimate of time-mean speed, either using direct measurements from double loop speed detectors ${ }^{2,3}$ or by the relationship between flow, speed and occupancy ${ }^{4}$ using single loops $s^{5,6,7,8,9}$, before attempting to estimate journey time.

Estimates of travel times on motorways have been achieved using stochastic probability models $^{9}$, statistical estimates of volume, occupancy and speed ${ }^{8}$, and time-mean speed measurements ${ }^{3}$. 
Estimates of travel times have also been achieved in urban signalised areas by identifying traffic signal phases and modelling the delay to queued vehicles passing over an upstream controlling detector ${ }^{6}$.

There is often considerable unexplained day-to-day variability in recorded journey times along the same stretch of road. The ability to train a neural network using examples of various road conditions might produce a more accurate and versatile journey time estimation tool compared to the more mechanistic time-mean speed approaches. Using flow and occupancy data related to actual measured journey times, techniques involving neural networks ${ }^{7}$ and fuzzy $\operatorname{logic}{ }^{5}$ have been used to estimate journey times on signalised links.

A technique enabling video footage to be collected in synchronisation with loop-occupancy data $^{10}$ has led to the development of detailed databases containing vehicle loop profiles matched to measured journey times. This allows the performance of various journey time estimation techniques to be assessed in detail. This paper describes the on-street performance of a new neural network based journey time estimator compared to a simplistic speed-based technique on a non-signalised road in Southampton.

\section{$\underline{\text { Objectives }}$}

Using a technique to derive 30-second estimates of mean vehicle speed, average loopoccupancy time per vehicle (ALOTPV), average time-gap between vehicles (ATGBV) and percentage occupancy from the digital output produced by single inductive loop detectors operating on a 250 -ms sampling cycle ${ }^{11,12}$, our aims were to:

a) Develop a mechanistic and a neural network model to estimate post-event journey times in Southampton along two non-signalised links fitted with single inductive loop detectors.

b) Collect digital loop-occupancy data in synchronisation with the measured journey times of vehicles travelling along the two links. 
c) Assess the accuracy of the journey time estimates produced by the models using:

i) Data from all detectors on the survey links.

ii) Data from pairs of detectors on the survey links.

\section{Concept Development}

The methodology for extracting the necessary parameters from the detector data has been described in detail ${ }^{11,12}$. Single inductive loop detectors buried in the road surface produce an analogue signal which is turned into a digital signal (0/1) by a detector pad usually located in the controller. A ' 1 ' indicates the presence of metal over the loop ${ }^{13}$. Cars sit closer to the road surface than vans and lorries and cut more lines of magnetic flux as they pass over a detector, so generating a higher inductance change.

The vehicle-presence status of a SCOOT-type detector ${ }^{14}$ is checked at 250 -ms intervals. The presence of a vehicle is indicated by a variable number of successive 1's, each 1 representing 250-ms of occupancy ${ }^{11}$. The number of 1's produced $(\mathrm{N})$ for a single vehicle is given by Equation 1.

$$
\mathrm{N}=4 *(\mathrm{DL}+\mathrm{VL}) / \mathrm{VS} .
$$
Equation (1)

Where:

$\mathrm{N}$ is the loop occupancy time of the vehicle (the number of digital 1's produced, each representing 250-ms of occupancy)

DL is the detector's effective magnetic length (metres)

VL is the effective magnetic length of the vehicle (usually metallic chassis length) (metres)

VS is the vehicle speed (metres/second) 
Previous research ${ }^{11}$ developed the parameters of ALOTPV and ATGBV to help describe traffic conditions over a detector. The ALOTPV for a 30-second fixed-time interval is obtained by taking the number of 250-ms occupancies and dividing by the number of vehicles. This was engineered to return a figure of between 1 and 120, the former indicating free-flow conditions, the latter stationary traffic.

ATGBV uses the same process for calculating ALOTPV but in reverse, (the number of 250ms vacancies divided by the number of vehicles) and produced a ratio between 1 and 120, the former indicating stationary traffic, the latter free-flow conditions. Regressing 30-second ALOTPV data against the mean measured speeds of vehicles (determined through video surveillance) gave reliable estimates of speed $^{12}$. All data were collected through the ROMANSE traffic control centre in Southampton ${ }^{15}$.

\section{$\underline{\text { Survey areas }}$}

Two links feeding Southampton city centre were fitted with single inductive loop detectors at approximately 100-metre intervals ${ }^{16}$. The A33 Bassett Avenue, (a four lane un-segregated Aclass road) had eleven loops fitted over 1149 metres (Figure 1a) and the A35 Winchester Road, (a two-lane A-class road) had nine loops fitted over 897 metres (Figure 1b). Data collection took place during the morning peak (07:30-09:30) over a three-week period in October 1996. 
Figure 1a. A33 Bassett Avenue Southbound inside lane detectors

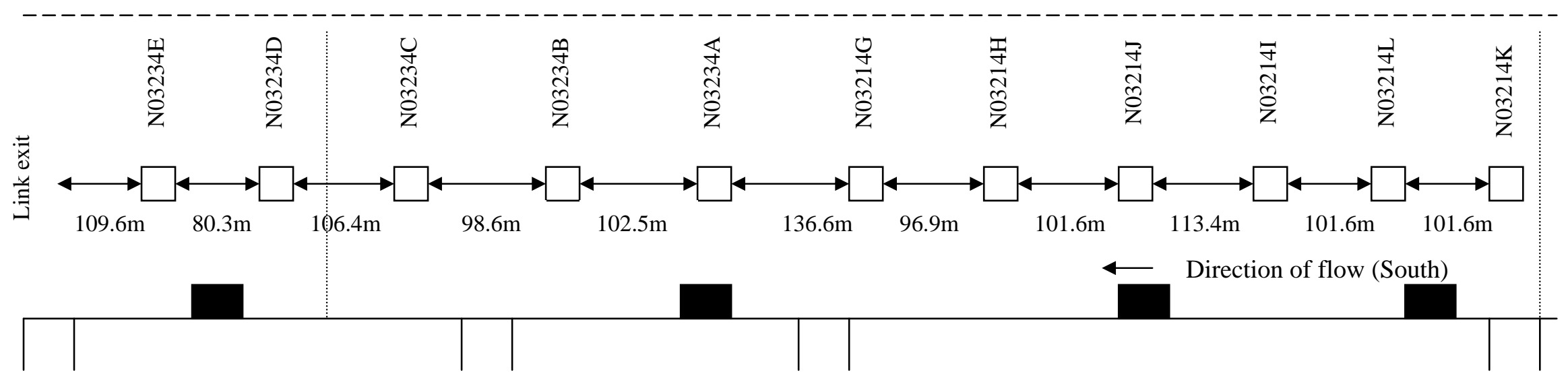

Figure 1b. A35 Winchester Road Southbound inside lane detectors

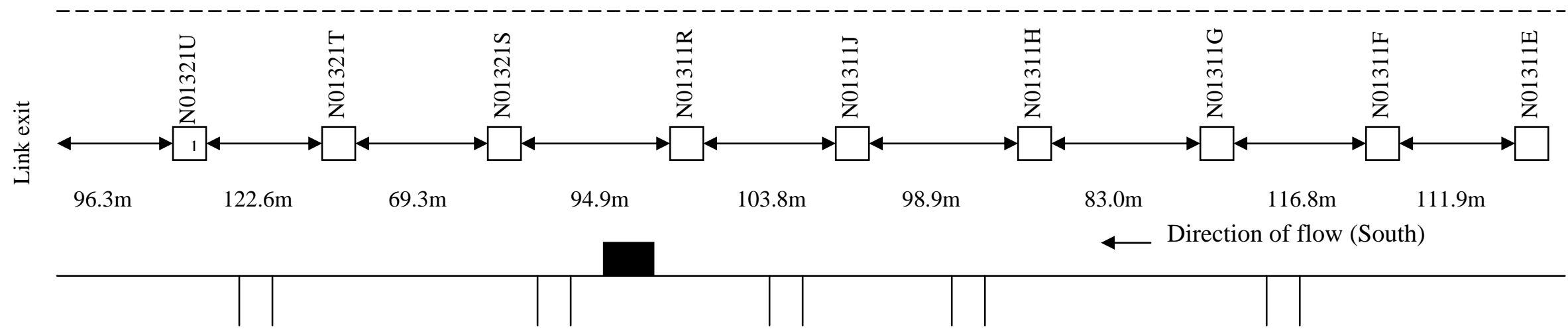

Bus stop $\quad$................ Pelican crossing $\quad \mid \quad$ Side road $\quad \square \quad$ Detector (i.d. number) 


\section{Observed link journey times}

Considerable variability was found between the observation days in the journey times derived from registration plate surveys (Figure 2). This demonstrated the potential difficulties involved in gathering a representative training sample of 'typical' days for a neural network journey time estimator.

Figure 2. The average measured journey times (seconds) of vehicles travelling South along the $1149 \mathrm{~m}$ of Bassett Avenue.

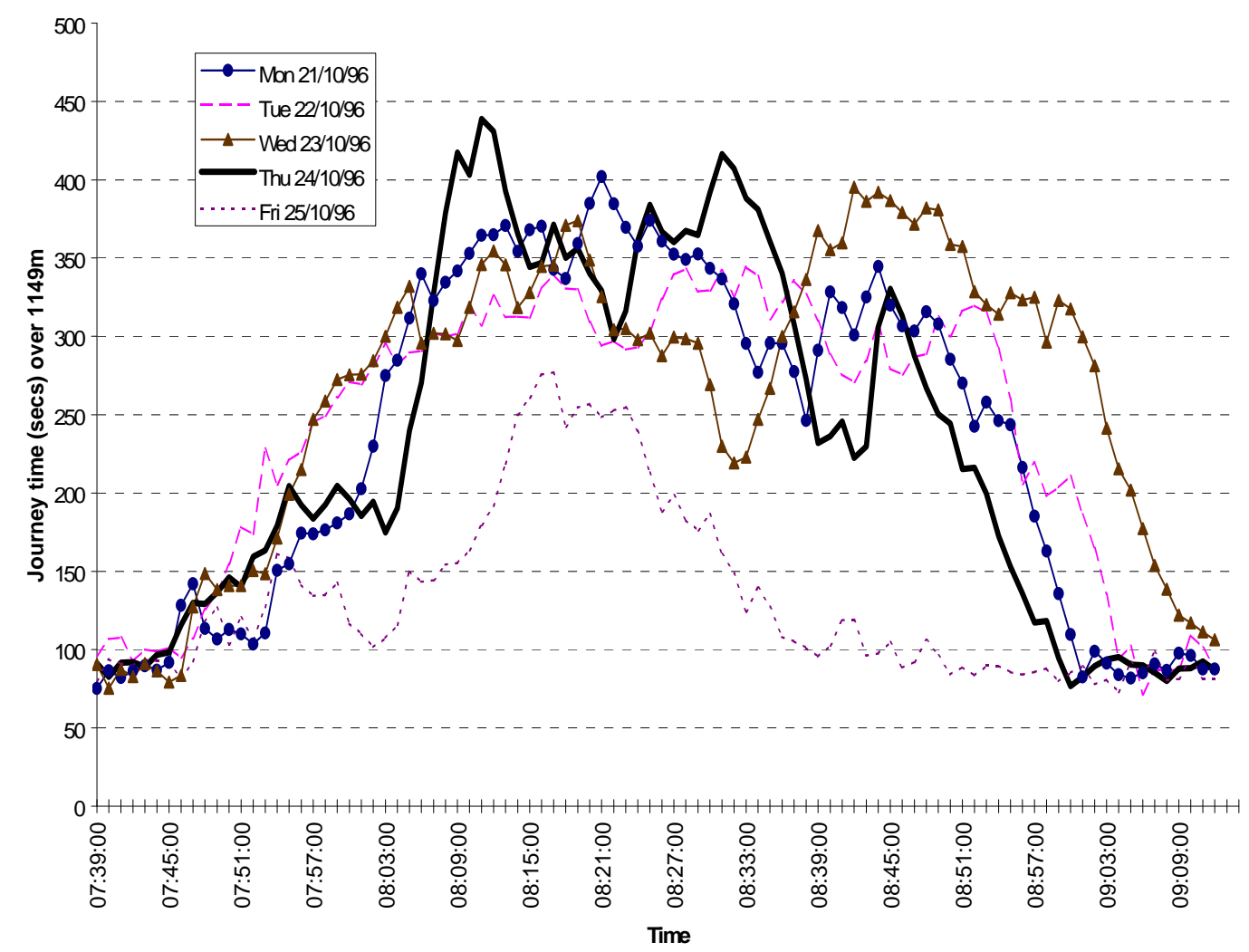




\section{Mechanistic journey time estimation technique}

The five stages in the mechanistic model were as follows:

Stage 1. For the first two detectors in the user-defined list, calculate the ALOTPV and average vehicle speed for the previous 30 -second period ${ }^{11,12}$.

Stage 2. Taking the mean speed of vehicles passing over both detectors ( 1 and 2$)$ in that period, calculate the arrival time at detector 2 according to the mean estimated vehicle speed and the distance to the next downstream detector (detector 2). (This assumes that vehicle speed is constant over the distance.)

Stage 3. When the 30-second projected arrival interval has elapsed at the downstream detector (detector 2), determine the ALOTPV and mean vehicle speed for detectors 2 and 3 at this time. According to the mean speed and distance to the next downstream detector (detector 3), calculate the arrival time at detector 3.

Stage 4. Continue this process until the end of the link is reached and allot the overall link journey time to the initial entry time interval.

Stage 5. Return to the first detector and repeat the whole process for vehicles entering the link during the second time interval.

The mechanistic approach assesses the mean speed of the traffic over each pair of detectors and estimates travel times between them according to distance and assumed constant mean speed. This is done on a historical 30-second basis. The effects of vehicles entering or leaving the road are taken into consideration in the 30 -second mean speeds produced by the detectors. If a vehicle decelerating to leave or accelerating to enter the major road causes other following vehicles to slow then this may well lower the mean speed recorded at an upstream 
detector. The longer the distance between detectors the greater the chance of speed variation and travel time error through the effects of bus stops, pelican crossings and side road entries. If in a theoretical situation, detectors were positioned up and down stream of a factory entrance, the overall mean speed derived might be higher than reality, due to the inability of the detectors to reflect the influence of slower vehicles entering and exiting the link between them.

\section{Neural Network journey time estimation technique}

The neural network models described here were built using NeuralWare's 'Predict' software ${ }^{17}$ which sits within Microsoft Excel. Data are fed into the Predict model builder using Excel spreadsheets. 'Predict' not only generates a neural network based on a set of data but manipulates, transforms and selects the data before they are used. During the data analysis faze, Predict takes each data field (in our case ALOTPV, ATGBV and percentage occupancy from each detector) and determines the types of transformations which work best in combination for predicting the desired output (in this case, journey time). Transformations used include identity function, exponential function, square function, inverse function and hyperbolic tangent function.

Predict's variable selection process then uses a genetic algorithm to identify subsets of the selected transformations which provide the closest matches to the target output.

The neural networks created were designed using an adaptive gradient learning rule which is a form of back propagation. Instead of using a fixed architecture for the design where the number of hidden processing layers are fixed, Predict uses a constructive method called 'cascade learning' to determine the optimum number of hidden processing layers. This can lead to different numbers of hidden layers being used between networks. 
Matching the vehicle registration plates extracted from video surveillance gave the overall journey times of the vehicles travelling down the two test sites. The basis for training was the ability to link the 30-second loop-occupancy data (ALOTPV, ATGBV and percentage occupancy) to the average measured journey times of the vehicles in each 30-second start interval (Table 1). Five days worth of measured journey times had been collected on both the Bassett Avenue and Winchester Road. Due to the day-to-day journey time variability observed it was decided in the first instance to give the networks a minimum of four days training data, testing on the remaining unseen day. (In the event, a detector fault on Bassett Avenue meant that only four days worth of data were available at this site and hence the relevant neural networks were only given 3 days worth of training data).

Table 1. An example of the training data used as inputs to a neural network journey time estimator. (For each 30-second interval the ALOTPV, ATGBV and percentage occupancy (\% Occ) values are shown at each detector site together with the average measured journey times of the vehicles in that period).

\begin{tabular}{|c|c|c|c|c|c|c|c|}
\cline { 2 - 7 } \multicolumn{1}{c|}{} & \multicolumn{3}{c|}{ Detector 1311E1 } & \multicolumn{3}{c|}{ Detector 1311J1 } & $\begin{array}{c}\text { Measured } \\
\text { Journey Time } \\
\text { (secs) }\end{array}$ \\
\hline $\begin{array}{c}\text { Start } \\
\text { Interval }\end{array}$ & ALOTPV & ATGBV & \% Occ & ALOTPV & ATGBV & \%cc Oc & 220 \\
\hline $08: 10: 00$ & 2.3 & 13.2 & 18.1 & 3.8 & 11.5 & 26.2 & 218 \\
\hline $08: 10: 30$ & 2.4 & 22.1 & 21.3 & 2.9 & 13.9 & 25.2 & 235 \\
\hline $08: 11: 00$ & 2.4 & 10.3 & 15.2 & 3.0 & 13.2 & 32.1 & 240 \\
\hline $08: 11: 30$ & 2.6 & 18.2 & 31.6 & 2.7 & 19.5 & 30.2 & 232 \\
\hline $08: 12: 00$ & 2.3 & 33.5 & 14.2 & 4.2 & 9.5 & 38.5 & \\
\hline
\end{tabular}

In summary, the neural network was trained by presenting it with a picture of the link conditions at each detector every 30-seconds, married to the measured journey times of the vehicles over the whole link who set off from the origin during that interval. During testing, an estimate of link journey time based on the immediate conditions was made every 30seconds. The effects of slower vehicles exiting or entering the link would be represented in 
the 30-second ALOTPV, ATGBV and percentage occupancy values of upstream detectors, if following vehicles were also forced to slow as a direct result.

Several networks were designed using various combinations of detectors and formats of training data for both the Winchester Road and Bassett Avenue. The accuracy of the journey time estimates produced were compared with those derived by the mechanistic model.

\section{A33 Bassett Avenue: Journey time estimations using all available detectors}

Previous work $^{12}$ demonstrated that ALOTPV was the most successful parameter for estimating speed over single inductive loops. A neural network was designed and trained using ALOTPV data from all the detectors in the inside lane (Figure 1a) and tested on the 22/10/96. The best results were obtained when using 23, 24 and 25 October 1996 as training examples in a 7-8-1 architecture (7 detector inputs passing through 8 hidden processing layers to produce 1 output). During the variable selection process, various transformations of the ALOTPV data were used and the output from 5 detectors (3214K, 3214L, 3214J, 3214G and 3234C) was rejected for not contributing significantly to the learning process. An internal correlation of 0.945 (reflecting the accuracy of the neural network's estimates of journey times in relation to the measured values) was the best score achieved by the network during the building process.

The results (Figure 3) showed that (using a paired t-test) there were significant differences between the mean journey time deviations derived by the two prediction methods $\left(t_{(170)}=5.8\right.$, $\mathrm{p}<0.001)$. On average the mechanistic approach significantly over-estimated journey times (27.5 seconds) compared to the neural network (5.1 seconds). This equated to a mean absolute percentage deviation from the mean measured journey time (MAPD) of $15 \%$ and $12 \%$ respectively. There were also significant differences between the variances given by the two prediction methods, $\left(\mathrm{F}_{(170,170)}=1.389, \mathrm{p}<0.01\right)$, the neural network approach giving 
significantly less variability from the mean measured journey time compared to the mechanistic estimator.

Figure 3. Measured journey times compared with model estimates over the $1149 \mathrm{~m}$ of the A33 Bassett Avenue (South bound inside lane) between the Chilworth and Winchester Road roundabouts, (22/10/96). The neural network was presented with data from all available detectors for training.

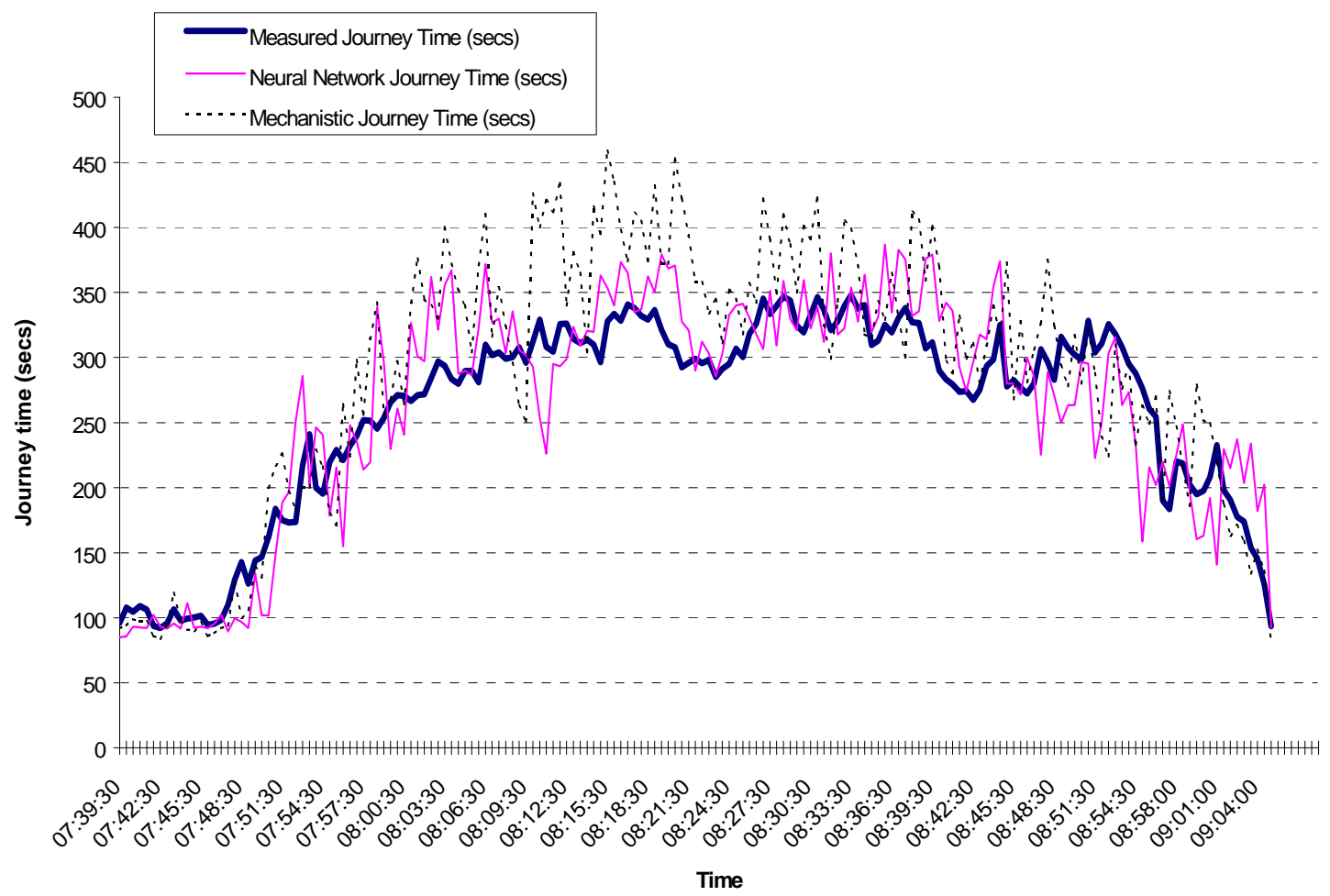

\section{A33 Bassett Avenue: Journey time estimations using only two detectors}

If single inductive loops were to be used for estimating journey times in any commercial application, funding would not be available to locate them every $100 \mathrm{~m}$.

In trials using pairs of detectors, the most accurate neural network estimates of the journey times over the whole $1149 \mathrm{~m}$ were obtained using detectors 3234A and 3234D 307m apart, and $652 \mathrm{~m}$ and 190m from the link entry and exit respectively. Detectors 3214K and 3234D gave the best mechanistic estimates over the 1149m. Figure 4 shows how the mechanistic 
approach frequently over and under estimated the journey time, being unable to gauge accurately the speed fluctuations between the two loop sites. The neural network was given the ALOTPV, percentage occupancy and ATGBV data for both detectors for 23, 24 and 25 October 1996. The ALOTPV from 3234A and the ATGBV and percentage occupancy from detector 3234D were rejected during the variable selection process. Testing during the building process gave an internal correlation of 0.89 using a 5-4-1 network architecture.

Figure 4. Journey time estimates over the $1149 \mathrm{~m}$ of the South bound inside lane of the A33 Bassett Avenue between the Chilworth and Winchester Road roundabouts, (22/10/96). The neural network was presented with data from only two detectors for training.

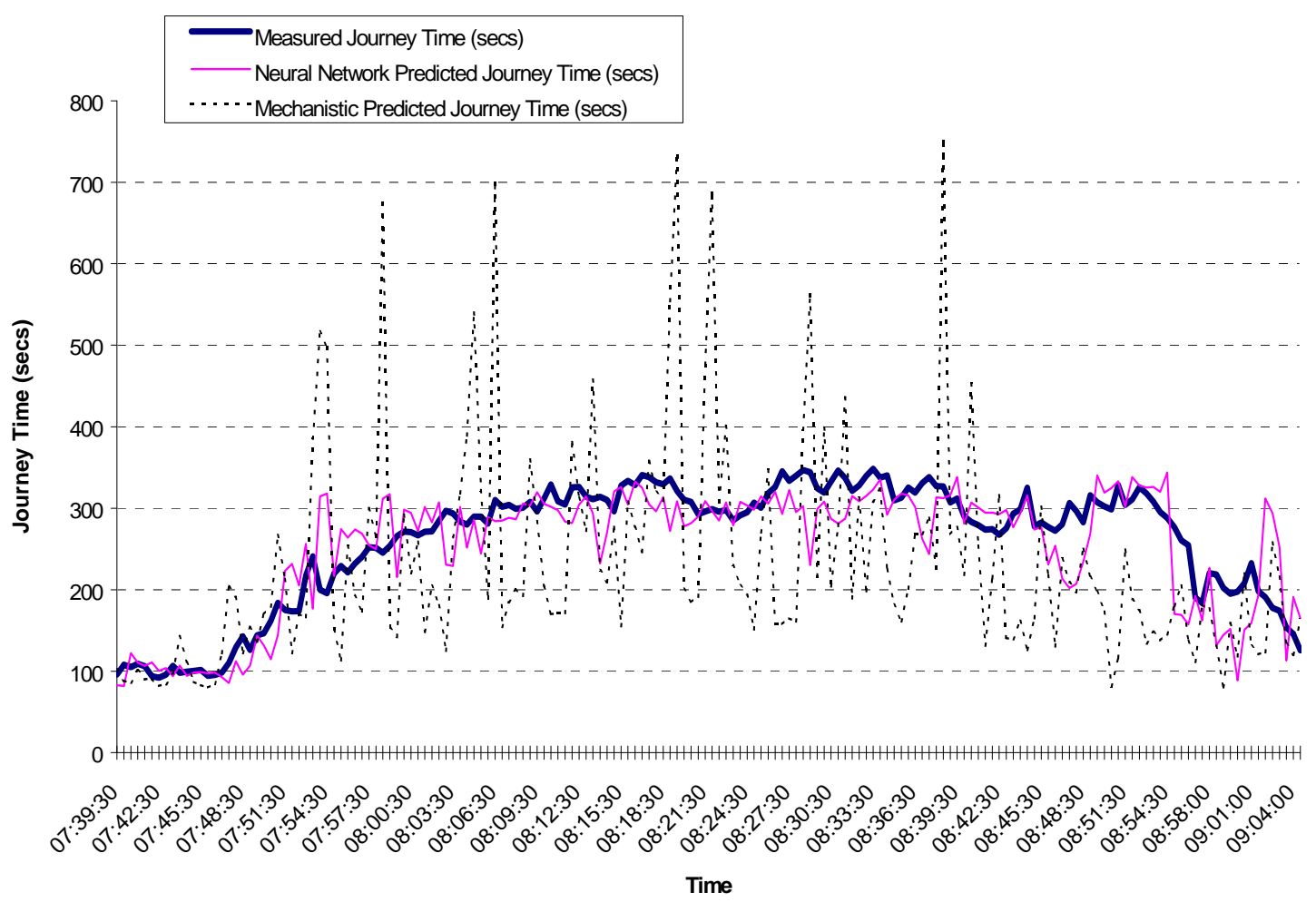

The results showed that on average the mechanistic approach under estimated journey times by 28.8 seconds (34.5\% MAPD) whilst the neural network under estimated the time by 8.8 seconds (12.8\% MAPD). A paired t-test showed that there were again significant differences between the two techniques in the mean deviations from the measured journey times, $\left(\mathrm{t}_{(170)}=\right.$ 
2.13, $\mathrm{p}<0.05)$. An F-test showed that there were also highly significant differences between the variances given by the two models, $\left(\mathrm{F}_{(170,170)}=8.67, \mathrm{p}<0.001\right)$, the neural network approach giving significantly less variability from the measured journey times compared to the mechanistic estimator.

Comparing the performance of the neural network created using data from all detectors against that using data from only two showed that there was no significant increase in the MAPD, (12\% and $12.8 \%$ respectively), $\mathrm{t}_{(170)}=0.28, \mathrm{p}>0.05$ although the use of only two detectors for training did lead to a significant increase in the variability from the measured journey times, $\left(\mathrm{F}_{(170,170)}=1.5, \mathrm{p}<0.05\right)$. The performance of the mechanistic approach was significantly impaired when data from only two detectors were used compared to all the detectors in terms of both MAPD (34\% compared to $15 \%$ respectively, $\mathrm{t}_{(170)}=8.3, \mathrm{p}<0.001$ ) and the variance around the mean $\left(\mathrm{F}_{(170,170)}=6.7, \mathrm{p}<0.001\right)$.

\section{A35 Winchester Road: Journey time estimations using all available detectors}

Five days of loop-occupancy and measured journey time data were collected along Winchester Road during the October 1996 surveys (Figure 1b). The A35 Winchester Road differed in character from Bassett Avenue in that parking was permitted along its entire length. Vehicles stopping to drop passengers or visit the shops at the Hill Lane end of the link frequently disrupted traffic flow.

Using all 9 detectors, a neural network was designed and trained on ALOTPV data from 7,9,10 and 11 October and tested on the 8/10/96. During the building process the data from detectors 1311E, 1311G, 1311J, 1321S were rejected for not contributing to the learning process. Through a 5-0-1 architecture, the neural network achieved an internal correlation of 0.96 during training and outperformed the mechanistic model during testing with a MAPD of $10.8 \%$ compared to $12.9 \%$. Figure 5 shows how the mechanistic estimator again suffered from greater inaccuracies during the peak period, an average over estimation of 17.7 seconds. 
A paired t-test and an F-test showed that there were significant differences in the mean deviations from the measured journey times and the variances around those means between the two techniques, $\mathrm{t}_{(228)}=7.86, \mathrm{p}<0.001$ and $\mathrm{F}_{(228,228)}=2.15$, $\mathrm{p}<0.001$.

Figure 5. Journey time estimates over the 928m of the A35 Winchester Road, South bound between the Winchester Road and Hill Lane roundabouts, (8/10/96). The neural network was presented with data from all available detectors for training.

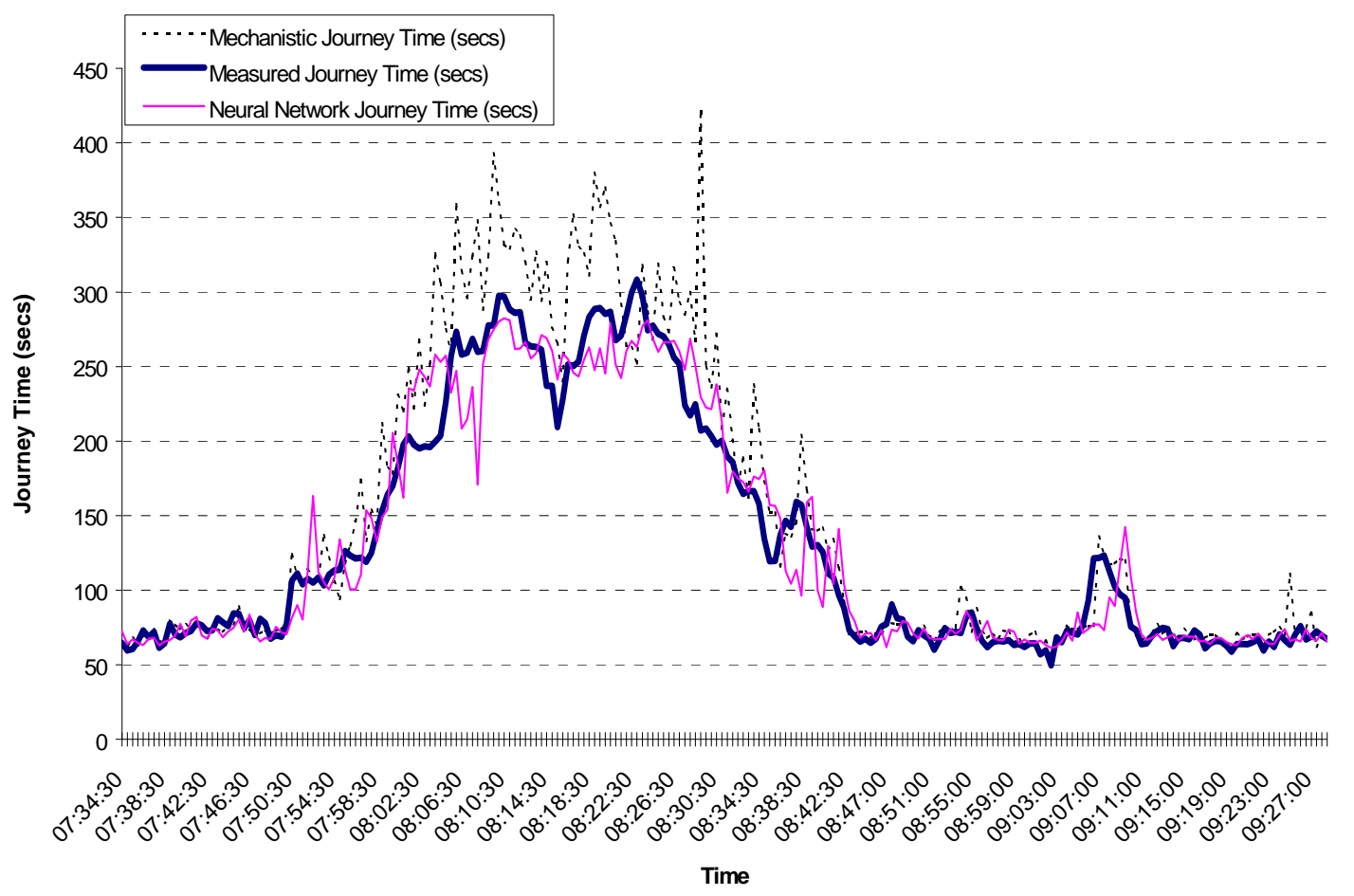

\section{A35 Winchester Road: Journey time estimations using only two detectors}

A neural network given ALOTPV, ATGBV and percent occupancy data only from detectors $1311 \mathrm{H}$ and $1321 \mathrm{U}$ for $7,9,10$ and 11 October 1996 was tested on the same output from the 8/10/96. Through a 3-0-1 architecture the network produced an internal correlation of 0.93 during training and the MAPD produced during testing was $13 \%$ compared to the mechanistic's 25\%, (Figure 6) the latter method over-estimating by an average 14 seconds. Again a paired t-test and an F-test showed that there were significant differences in the mean 
deviations from the measured journey times and in the variances around those means between the two models, $\mathrm{t}_{(228)}=2.84, \mathrm{p}<0.05$ and $\mathrm{F}_{(228,228)}=5.6, \mathrm{p}<0.001$.

Figure 6. Journey time estimates over the 928m of the A35 Winchester Road, South bound between the Winchester Road and Hill Lane roundabouts, (8/10/96). The neural network was presented with data from only two detectors for training.

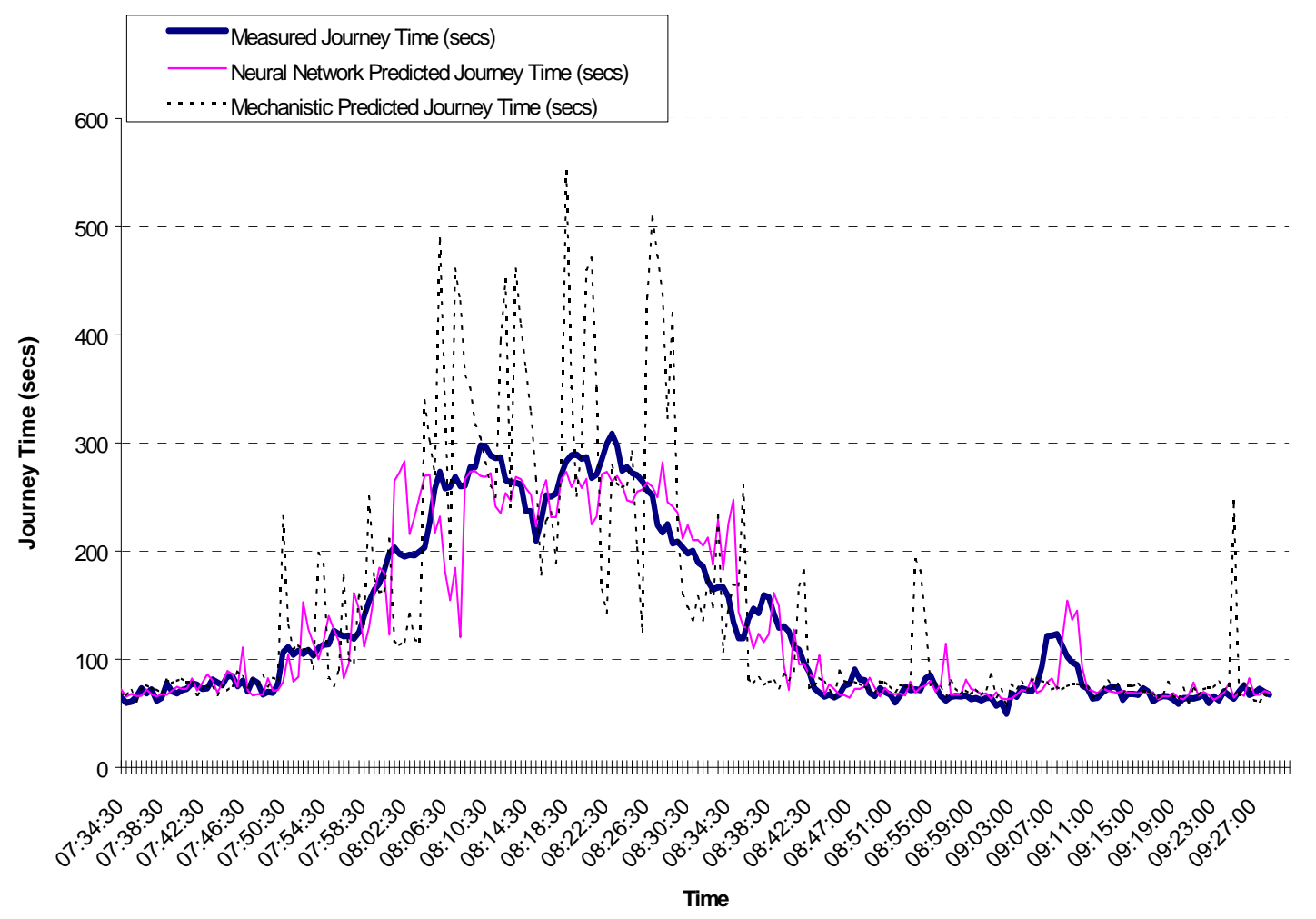

Comparing the performance of the neural network created using data from all detectors against that using data from only two showed that there was a significant increase in the MAPD (10.8\% compared to $13 \%$ respectively), $\mathrm{t}_{(228)}=3.39, \mathrm{p}<0.001$ and in the variability from the measured journey times, $\left(\mathrm{F}_{(228,228)}=1.78, \mathrm{p}<0.001\right)$. The performance of the mechanistic approach also degraded significantly when data from only two detectors were used compared to all the detectors in terms of MAPD (25\% compared to $13 \%$ respectively, $\left.\mathrm{t}_{(228)}=6.67, \mathrm{p}<0.001\right)$ and the variance around the mean $\left(\mathrm{F}_{(228,228)}=5.92, \mathrm{p}<0.001\right)$. 


\section{$\underline{\text { Discussion }}$}

The accuracy of the ALOTPV derived speed estimates used in the mechanistic approach depended critically on a detector's level of sensitivity, relative to that of the loop on which the speed estimates had been originally determined ${ }^{12}$. Large variations in sensitivity were found between the detectors fitted on both the A33 and A35, affecting the loop-occupancy profiles of the vehicle platoons passing over them. This problem could be overcome if the sensitivity of each detector was set to the same level during installation.

Unlike the neural network technique, the mechanistic approach does not require the collection of training data. Although proved to be less accurate, it is much more flexible in being able to provide estimates using multiple combinations of differently spaced detectors. The neural networks designed in this research are unique to the links on which they were trained, containing the unique characteristics of the particular road (bus stops, pedestrian crossings and key junctions). A separate issue not addressed here is how conditions on a road change over time and how representative of 'typical' link conditions are the data that have been collected for training? How often would new training data be required to 'update' a network in order to keep pace with changing traffic conditions over time?

Collecting training data using registration plate surveys is an expensive process, and a key issue is the minimum amount needed for the training process. Networks can be over trained when so many examples are provided that estimates follow a mean trend line through the $\operatorname{data}^{18}$. Using the same two detectors which returned the most accurate journey time estimates on Bassett Avenue (3234A and 3234D) a neural network was designed, training on data from Tuesday 22/10/96 only (Figure 2.) The results showed that one-day’s training data were not enough to cater for the variation in congestion patterns between days. The MAPD were 28\% (Wednesday 23/10/96), 31\% (Thursday 24/10/96) and 49\% (Friday 25/10/96.) The lack of congestion on Friday 25/10/96 (Figure 2) was in complete contrast to that experienced on the training day leading to the poor result. 
The neural networks described here provide an estimate of journey time based on the ALOTPV, ATGBV and percentage occupancy values provided by a series of detectors in a given 30-second period. During peak periods, vehicles can often take up to 8 minutes to travel along the $1149 \mathrm{~m}$ of the A33 Bassett Avenue. In this situation the neural network provides a 'prediction' of journey time based on its training examples. This can be useful for traffic control room operators in monitoring and anticipating the changing status of the network.

Using on-line ALOTPV data collected from the ROMANSE traffic control centre, a real-time display showing 30-second updates of estimated journey times from four new neural networks has recently been developed (Figure 7). This has enabled the identification of abnormal traffic conditions, during both peak and off-peak periods. An example of this occurred on the $27^{\text {th }}$ June 2001 when an accident partly blocked the Southbound carriageway of the A33 (Figure 1a). All four neural networks used in this test (one trained solely on 1996 ALOTPV data, one on new data from 2000 and 2001, one on a mixture of 1996 and 2000 data and one training on all the ALOTPV data collected) correctly identified the accident which happened at 08:07. 
Figure 7. Journey time estimates over the $1149 \mathrm{~m}$ of the South bound inside lane of the A33

Bassett Avenue between the Chilworth and Winchester Road roundabouts, (27/06/01). The image is a screen-shot taken from a prototype journey time estimation operator interface.

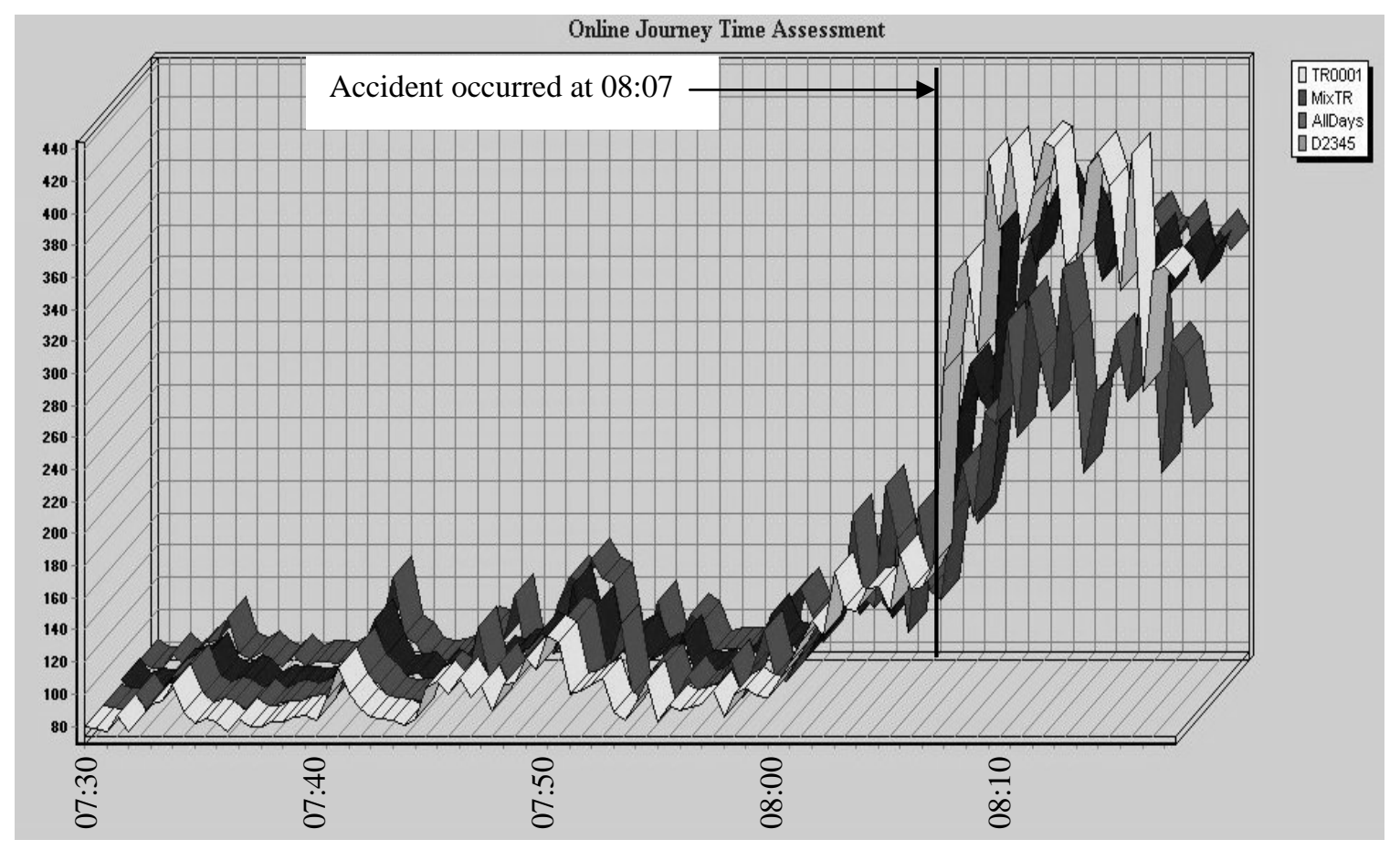

$\mathrm{X}$ Axis $=$ Time (30-second updates)

Y Axis = Journey Time (seconds)

'TR001' = A neural network trained on ALOTPV data collected during 2000 and 2001

'MixTR' = A neural network trained on ALOTPV data collected during 1996 and 2000

'All Days' = A neural network trained on all ALOTPV data collected

'D2345' = A neural network trained on ALOTPV data from 1996

\section{Conclusions: Potential uses for accurate link journey time forecasts}

The results showed that journey time estimates on non-signalised links using neural networks trained on three days of morning peak data give significantly greater accuracy compared to a mechanistic speed based approach. This was particularly evident when using the minimum of two detectors, (13\% MAPD compared to 25\% in the case of the Winchester Road).

There is great potential for this type of detailed, immediate post-event information. If journey times on non-SCOOT controlled links can be coupled with similar estimates through SCOOT controlled urban areas, complete routes could be monitored continuously. This has benefits 
both in terms of more accurate network management, (signal control, monitoring special events, incident detection) and in providing accurate driver information.

The accuracy of the estimates produced using pairs of detectors are encouraging as infrastructure costs would be reduced if only limited new detectors had to be installed. The traffic control room operator would benefit if journey time estimates could be obtained for areas of the network outside CCTV control. Real-time estimates of journey time would help identify abnormal congestion and aid the operator in deploying effective transport management strategies.

This work has used the 250-ms digital loop-occupancy data from single inductive loop detectors to determine journey times. Where available, similar output from other detector types could be used to provide direct speed measurements or values of flow and occupancy. 


\section{Bibliography}

1. Billington P (1997). Covering the network. Breaking the licence plate recognition costbarrier. Traffic Technology International. October/November issue. 28-32.

2. Morin J, Fevre R (1997). Real-time estimation of travel times on inter-urban motorways. $8^{\text {th }}$ 'International Federation of Automatic Control' Symposium, International Federation of Automatic Control (IFAC) Austria. Vol 3, 1193-1198. 16-18 June, Chania, Greece.

3. Van-Grol H (1997). Evaluating the use of inductive loops for travel time estimation. $8^{\text {th }}$ 'International Federation of Automatic Control' Symposium, International Federation of Automatic Control (IFAC) Austria. Vol 3, 1187-1192. 16-18 June, Chania, Greece.

4. Hall F, Persaud B (1989). Evaluation of speed estimates made with single-detector data from freeway traffic management systems. Transportation Research Record, 1232, 9-16.

5. Sisiopiku V, Palacharla P, Nelson P (1994). Fuzzy reasoning model for converting loop detector data into travel times. ISATA Advanced Transport Telematics Conference, Aachen, Germany, October.

6. Anderson J (1997). Travel time prediction in urban road networks. $8^{\text {th }}$ 'International Federation of Automatic Control' Symposium, Vol 3, 1181-1186. 16-18 June, Chania, Greece.

7. Cohen S, Nouveliere C (1997). Travel times on urban controlled links: A neural network approach. Proceedings of the $4^{\text {th }}$ World Congress on Intelligent Transport Systems, 21-24 October, ICC Berlin, Germany.

8. Dailey D (1997). Travel time estimates using a series of single loop volume and occupancy measurements. Proceedings of the $76^{\text {th }}$ Annual Meeting of the Transportation Research Board, January 12-16, Washington, D.C. Paper No. 97-0378.

9. Petty K, Bickel P, Jiang J, Ostland M, Rice J, Ritov Y, Schoenberg F (1997). Accurate estimation of travel times from single loop detectors. Proceedings of the $76^{\text {th }}$ Annual Meeting of the Transportation Research Board, January 12-16, Washington, D.C. Paper No. 97-1043.

10. Cherrett T, Bell H, McDonald M (1995). The Measurement of Speed, Journey Time and Queue Status on non SCOOT controlled links, I.E.E. Colloquium Digest, Professional Group C12 (Transport electronics and control) "Urban Congestion Management", 5, 15.

11. Cherrett T, Bell H, McDonald M (2000). Traffic management parameters from single inductive loop detectors. Transportation Research Record 1719, National Research Council, Transportation Research Board.

12. Cherrett T, Bell H, McDonald M (2001). Estimating vehicle speed using single inductive loop detectors. Institute of Civil Engineers, Transport Journal, paper number 12371.

13. Pursula M, Kosonen I (1989). Microprocessor and PC-Based Vehicle Classification Equipment Using Induction Loops. Second international conference on Road Traffic Monitoring, IEE, London. 1989. pp. 24-28.

14. The Department Of Transport (1995). The 'SCOOT' Urban Traffic Control System. The Department of Transport Traffic Advisory Leaflet, DETR, London. April. 
15. Wren A, Jones P (1996). ROMANSE - Road Management System for Europe. $8^{\text {th }}$ International Conference on Road Traffic Monitoring and Control, IEE, 23-25 April, Publication No. 422, 23-27.

16. Richards A, Mcdonald M, Shinakis E (1994). The use of SCOOT loops on non-signalised links. IFAC $7^{\text {th }}$ Symposium on Transportation Systems, Tianjin, China, 1994.

17. NeuralWorks Predict (1995). NeuralWare, Inc. Technical Publications Group, 202 Park West Drive, Pittsburgh, PA 15275.

18. Lyons G (1994). The application of artificial neural networks to model driver decisions. Ph.D. Thesis, Division of Civil Engineering, School of Engineering, University College of Wales, Cardiff. 